\title{
Specification-driven Performance Monitoring of SDL/MSC-specified Protocols
}

\author{
P. Dauphin, W. Dulz, F. Lemmen \\ University of Erlangen-Nürnberg \\ IMMD VII, Martensstr. 3, 91058 Erlangen, Germany, Fax: +49-9131/85-7409, \\ email: $\{$ dauphin, dul $\mathrm{z}$, lemmen\}@informatik.uni-erlangen.de
}

\begin{abstract}
Protocol testing implies the functional analysis of a given implementation under test as well as its temporal performance evaluation. If protocols are formally specified sophisticated techniques and tools exist for analyzing functional properties, e.g. finding deadlocks or livelocks. Methods, however, for the temporal performance evaluation of formally specified systems are still in their infancy and tools for performance monitoring have to be developed.

In the telecommunications industry SDL and MSC specifications are common FDTs for developing reactive real-time systems. While SDL specifications describe the overall system architecture in detail, MSC scenarios are used to specify the load-dependent behavior of interacting system components.

For SDL/MSC specified systems, we present a method which allows a systematic quantitative temporal analysis of such systems. The basic idea is to apply MSCs in order to concentrate on the main parts of interacting SDL processes such as signal exchanges and timeouts. Thus, we can restrict the monitoring overhead by focussing on the most important points of interest in the interaction of communicating processes, and evaluating their behaviors by means of monitored event traces. Our approach allows us to integrate software and performance engineering techniques into a comprehensive methodology. A prototype tool called MISS was developed to proof the suitability and practicability of the method.
\end{abstract}

\section{Keywords}

Event-driven Monitoring, Message Sequence Chart (MSC), Performance Engineering, Specification Description Language (SDL), Specification-driven Monitoring 


\section{INTRODUCTION}

Due to the ever increasing complexity of parallel and distributed systems integrated development and analysis tools which cover the whole life cycle of the system must be provided. The classic waterfall model for systems and protocol engineering defines all steps and phases which are necessary for managing the system's evolution, such as requirement analysis, design and specification, implementation, test and monitoring of the real system.

For reactive real-time systems like communication systems it is necessary to define models and to use paradigms which simplify the view of a complex system and which concentrate the attention on the development and analysis of the main parts of the system. Formal description techniques (FDTs) provide a unifying theoretical basis and permit

- to characterize the system behavior by abstractions such as creation and termination of concurrent parallel processes, sending and receiving of synchronizing messages between cooperating processes, setting and resetting of timeouts and transition conditions between different system states,

- to construct dedicated development tools such as graphical editors and user front-ends, functional and temporal validators, simulators, compilers, monitor and evaluation systems that support each step of the development process,

- to develop modular and hierarchical systems by reusing less complex subsystems with simpler functional or temporal properties,

- and last but not least to apply these techniques to a wide range of engineering problems.

An open issue concerning FDTs, however, is the development of methods and tools for studying the performance of systems which are specified and implemented by using an FDT approach. The performance evaluation starting from an abstract system model either can be achieved by mathematical analysis and simulation techniques in the design phase or by monitoring parts of the real system in the implementation and test phases. Because FDT environments support the automatic generation of executable code from a given formal system specification, monitoring tools can reuse the same specification as a formal monitoring model. One of the most promising ways of gaining insight into the temporal behavior of a system is event-driven monitoring, a technique which allows to construct event traces by monitoring the execution of real applications and selecting only those events which are related to interesting system states. The events are defined by inserting monitoring instructions into the program under investigation (program instrumentation). The monitoring instructions write event tokens to a hardware interface which is accessible for a hardware monitor (hybrid monitoring) or into a reserved memory area (software monitoring). Defining events with program instrumentation each monitored event token can be clearly assigned to a point in a program. This provides a problem-oriented reference. Thus, the evaluation of the event trace yields insight at the program level which is familiar to the program designer.

The main problems of event-driven monitoring, namely what to measure and where to find relevant instrumentation points inside the source program are answered implicitly using the 
technique of model-driven monitoring [Qui93, HQ93, $\mathrm{HKM}^{+}$94]. In model-driven monitoring the event, a common abstraction of event-driven monitoring and event-oriented models like graph models, Petri nets, queueing models, etc., is used to integrate modeling and monitoring. A so-called monitoring model is derived from the problem specification considering the aim of measurement. This monitoring model forms the basis for both the instrumentation of the program (model-driven instrumentation) and the evaluation of an event trace (model-driven evaluation) which is monitored during the execution of the instrumented program.

Formal monitoring models allow us to use monitoring procedures in a systematic and automatic way. The main disadvantage of model-driven monitoring, however, is the informal generation of the monitoring model, i.e. in general the model can not be derived automatically and may therefore be error-prone which means that the implementation and the monitoring model can be inconsistent [DKQ92].

Because model-driven monitoring is independent of the modeling technique used, FDT specifications may also serve for generating the monitoring model. Using the formal specification as monitoring model let us overcome the disadvantage of model-driven monitoring, that is the implementation is consistent with the monitoring model. We call this method specification-driven monitoring.

In this paper, we present the method of specification-driven monitoring by using the FDT technique SDL in conjunction with MSC. System design with SDL/MSC is reviewed in the next section. Section 3 describes the new method for MSC-based monitoring of SDL specifications. In section 4 the method is applied to the performance monitoring of the INRES protocol. Finally, in section 5 we review the key benefits of specification-driven monitoring.

\section{SYSTEM DESIGN WITH SDL AND MSC}

Among the most popular and widely accepted FDT techniques we can distinguish between the three standardized approaches: LOTOS [BB89], which is based on a process algebra with its origin in MILNERs CCS calculus; ESTELLE (ISO 9074) and SDL (ITU Z.100, [CCI92]), both of which describe the system behavior by communicating extended finite state machines. While process algebras are better suited in describing functional properties by embedded algebraic laws and logic inference techniques, finite state machines are more intuitive for decomposing a system into automata, state graphs and transition tables. The main advantage of the high-level specification language SDL compared to ESTELLE is the possibility to either use the userfriendly graphical SDL/GR syntax or the equivalent SDL/PR notation which is better suited for the integration of machine-oriented tasks and design tools. Besides, SDL is also a commonly accepted specification and description language in the telecommunications industry for the development of protocol architectures and communication systems.

While SDL allows the specification of architectural aspects by decomposing a system into a hierarchy of blocks and channels which consist of parallel processes and signal routes, the recently standardized MSCs (Message Sequence Charts, ITU Z.120) are well suited to describe the dynamic behavior of concurrent processes. 
Each process is represented by a sequence of send and receive events which are logically ordered in an instance axis of the MSC diagram. It is also possible to define local and global process states which reflect major synchronization points between cooperating processes. For an example see Figure 4. The "method for system development based on MSC, SDL and functional decomposition" which is part of the "SDL Methodology Guidelines" in the appendices of Z.100 ([IT94]) describes the hierarchical top-down partitioning of a given system into lower-level subsystems until the process level is reached, thereby using SDL specifications and corresponding MSC scenarios.

Big efforts have been made recently to supply techniques and tools for SDL CAPE (ComputerAided Protocol Engineering) environments which provide automatic protocol validation, e.g. the formal proof that a given protocol specification is free of deadlocks or livelocks and does not contain unspecified message arrivals [Liu89], and directly produce implementations from given SDL specifications. A good overview of the state of the art can be found in [TOP94] which documents parts of the European TOPIC (Toolset for Protocol and Advanced Service Verification in IBC Environments) project.

Following [Liu89], the domain of protocol engineering is

- to provide a formal protocol specification by means of an appropriate FDT,

- to validate the functional correctness of the specification,

- to obtain some early indication of the expected efficiency,

- to compile major parts of the implementation directly from FDT specifications and finally

- to test the resulting implementation with respect to conformance, performance and reliability.

Most SDL CAPE environments like SDT [Tel93] or GEODE [GEO94] provide tool support for the functional validation, e.g. exploration of behavior trees in order to find system states which are involved in deadlock or livelock situations, but do not allow to explore temporal or performance aspects of SDL specified systems. Using SDL specifications as monitoring models allow us to integrate the method of model-driven monitoring in the SDL CAPE development cycle and thus, to remove the lack of performance evaluations tools for SDL specified systems.

\section{MSC-BASED MONITORING OF SDL SPECIFICATIONS}

Let us assume that the system under study (SuS) is totally specified in SDL and that various MSC scenarios are available which specify parts of the communication behavior within the SuS. One MSC together with the SDL system cover all the properties a monitoring model has to support for model-driven monitoring: the MSC indicates what to measure, whereas the SDL system shows where to instrument the SuS in order to get the desired insight into the dynamic behavior. The measurement preparation, during which the aim of the measurement is specified, consists of selecting a suitable MSC. Such an MSC which is consistent with the SDL specification of the SuS can be gained in numerous ways: 
- The MSCs is derived in the requirement definition phase of the SDL/MSC software development cycle (see section 2).

- The MSC is constructed interactively by stepping through the description of the SDL system by means of an SDL editor.

- The MSC is generated while simulating the SDL system triggered by given signals, timeouts, and the values of internal state variables to enforce the desired behavior of the SDL system.

- The MSC is constructed from the reachability tree which was built up in the validation phase of the SDL system.

- The HASSEWithMSC tool (see section 4) was used to generate an MSC from a measured event trace.

In the following sections, we present a new technique which uses an SDL/MSC specification to carry out monitoring in a systematic way. The approach which applies the ideas of specification-driven monitoring is implemented in the tool MISS (MSC-based Mon/toring of SDL-Specifications). MISS supports the monitoring process during the event selection, the program instrumentation, the measurement, and the analysis of a measured event trace.

\subsection{MSC-based measurements of SDL systems}

In order to prepare and execute the measurement of an SuS three tasks have to be carried out: event selection, program instrumentation, and the measurement itself. Figure 1 summarizes our approach for MSC-based measurements of SDL systems.

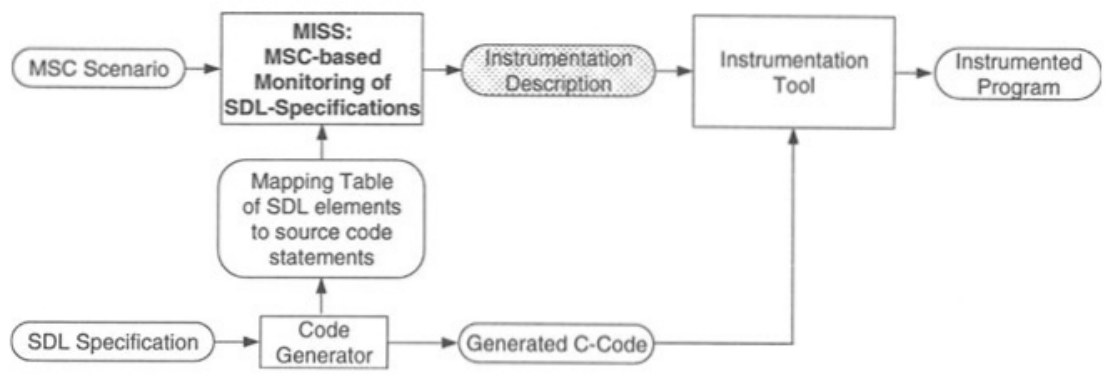

Figure 1 Generating an instrumented program from a given SDL/MSC specification.

\section{Event selection:}

The event selection is directly correlated with the amount of data to be collected during the measurement. To reduce the amount of data only the points of interest (PoI) should be selected for monitoring. Looking at an SDL/MSC-specified SuS PoIs can be found on three different levels of abstraction: With respect to the source code level each statement is a possible program 
point of interest (PPoI); concerning the SDL specification level each SDL element represents one possible $S D L$ point of interest (SPoI); last but not least MSC elements in the MSC specification represent possible $M S C$ points of interest (MPoI). Because an MSC specification focusses on the communication behavior which only reflects dynamic aspects of the complete SDL specification, and because each SDL element leads to numerous statements in the source code during program generation, the following relations between the different sets of PoIs hold:

number-of-PPoIs $\gg$ number-of-SPoIs $\gg$ number-of-MPoIs

Eq. (1) shows a reduction of the complexity adhered to measurements: On the one hand, the number of points to be instrumented as well as the amount of data monitored will be limited by MSC-based monitoring. On the other hand, the MSC serves to focus on application-specific PoIs, and thus, supports the event trace analysis and the interpretation of evaluation results. In using an MSC for instrumentation, only the MPoIs indicating where to instrument the program are considered. The interdependences of MPoIs which are specified in the MSC are also used in MSC-supported event trace evaluation (see section 3.2). Therefore, an MSC scenario which is a MISS input structure cannot only be an ordinary set of MSCs, but also has to define relations between the MSCs of the MSC scenario in order to guarantee the desired communication behavior of the SuS. Well-defined relations are the decomposition, repetition, concatenation, choice, and parallelization of MSCs as described in the "method for system development based on MSC, SDL and functional decomposition" [IT94].

To use MSC-based event selection in the framework of specification-driven monitoring, a one-to-one mapping between the MPoIs and the PPoIs must be found. Because MSC and SDL specifications of an SDL/MSC-based monitoring model are consistent, a first straightforward one-to-one mapping between MPoIs and SPoIs is directly given. A second one-to-one mapping between SPoIs and PPoIs is generated by the source code generator which transforms the SDL specification automatically into compilable source code. This second mapping, however, is hidden by the transformation process.

In order to produce the second one-to-one mapping, the source code generator must reveal internals of the mapping of any given SDL elements to its corresponding source code statements (see Figure 1). Although an explicit assignment table would be the most convenient way to do this kind of mapping, SDL CAPE tools normally do not offer it. Instead, the following methods exist to define an implicit relation between SDL elements and corresponding source code statements:

- mapping of each SDL element to the line number inside the source code,

- mapping of each SDL element to the identifier of a specific procedure, and

- mapping of each SDL element to an unambiguous annotation, which is some kind of source code comment.

The selection of the method depends on the SDL/MSC CAPE tool used. Because we built the MISS prototype in conjunction with SDT (SDL Design Tool) [Tel93] the third method was appropriate. 


\section{Instrumentation:}

By using MPoIs MISS produces an instrumentation description (see Figure 1). For each instrumentation point, the instrumentation description contains a command which specifies

1. which annotation has to be instrumented, i.e. where to insert a monitoring statement, and

2. which monitoring statement must be inserted, that is a $\mathrm{C}$-function which writes measurement events to a hardware interface or reserved memory areas.

We used the syntax and semantics of the instrumentation description language defined for the instrumentation tool AICOS (Automatic Instrumentation of $C \operatorname{prOgramS}$ ) [DHK ${ }^{+}$93].

\section{Measurement:}

The instrumented program must be compiled into an executable program. During the linking process the definitions of inserted monitoring statements (C-functions) have to be delivered. As MISS generates the instrumentation description, it exactly knows which bytes to send to the monitoring interface. Thus, in the case of software monitoring, the definition of monitoring statements can be generated by MISS automatically. In the case of hardware monitoring this function must be supplied by a linkable library.

During its execution each process of the instrumented SDL application produces a local event trace. If the local event traces are recorded by means of a common time basis*, they can be merged into a global event trace using the common time stamps in the local event traces for ordering. MISS generates a Unix shell script which carries out the merge.

\subsection{MSC-based event trace analysis of SDL systems}

For the analysis of measured event traces various tools are known, e.g. ParaGraph [HE91], TOPSYS [BR93] and TraceView [MHJ91]. We decided to use the universal monitor- and object-independent event trace evaluation environment SIMPLE (Source-related Integrated Multiprocessor and -computer Performance evaluation, modeLing, and visualization Environment) [Moh91]. The universality and independability of SIMPLE is achieved by

1. the event trace description language TDL [Moh92] which allows to describe the format of an arbitrary event trace in a problem-oriented way, and

2. tool configuration files which enables the adaption of each tool to the analysis of arbitrary object systems.

Both, the event trace description and the configuration files can automatically be generated by MISS given an MSC applied for the event selection (Figure 2). The problem-oriented TDL

\footnotetext{
*This can be achieved by synchronizing the distributed computers via the Network Time Protocol (NTP) [Mil94] or by carrying out the measurement using a distributed hardware monitor like $\mathrm{ZM} 4\left[\mathrm{HKM}^{+} 94\right]$ which has a global clock.
} 
event trace description is derivable due to the one-to-one mapping between MPoIs and PPoIs. The generation of tool configuration files depends on the aims of the measurement study.

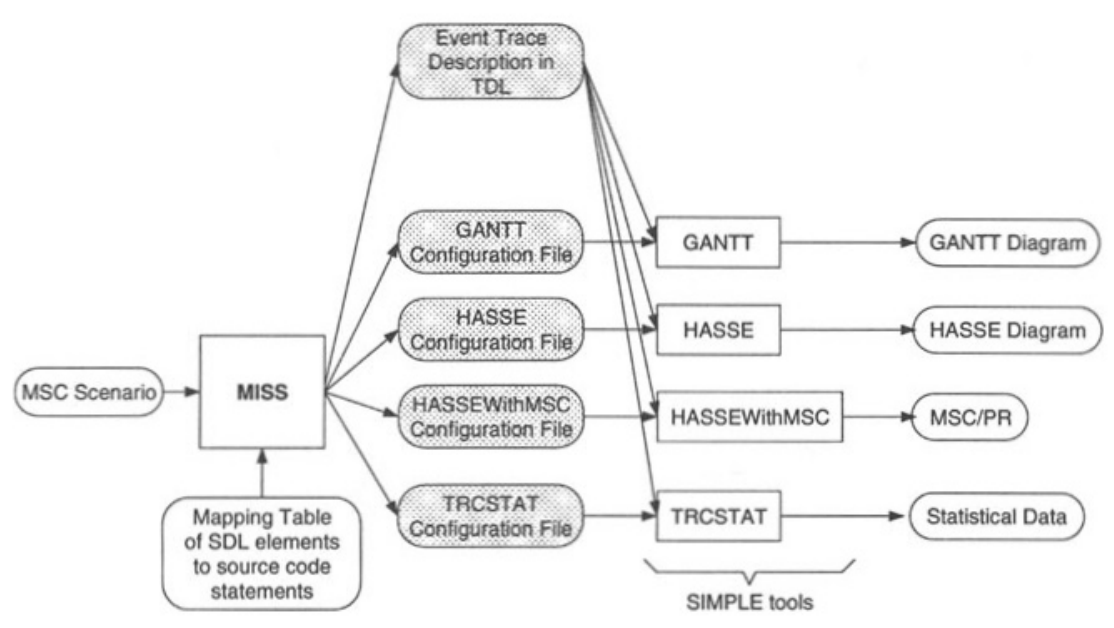

Figure 2 MSC-based trace evaluation with MISS and SIMPLE tools.

Event trace analysis is divided into validation where the consistency between the measured event trace and the corresponding SDL/MSC specification is checked, and performance analysis of the measured event trace which yields in various kinds of user-friendly diagrams (see Figure 5).

\section{Validation:}

From a given SDL specification an executable program only can be generated up to a certain degree - approximately $60 \%$ - by means of automatic procedures. The remaining part, called SDL environment, has to be provided by the user and therefore, is error-prone. Hence, an additional consistency check between the SDL/MSC specification which is the monitoring model and the measured event trace is necessary although all intermediate transformation steps are done automatically.

First, HASSEWithMSC generates an MSC from the measured event trace. The configuration file for HASSEWithMSC is yielded by MISS after interpreting causal dependences and state transitions provided in the SDL/MSC monitoring model. Then, the consistency between the derived MSC and the given SDL specification is validated using CAPE tools like SDT [Tel93] or GEODE [GEO94]. Note, that the validation should be performed before any other event trace analysis in order to be sure that the gained performance analysis results are meaningful. 


\section{Performance analysis:}

For recognizing causal relationships HASSE is used. The PPoIs which are necessary for the generation of the HASSE configuration file are the setting, resetting, and expiring of timers, the sending and receiving of messages, and the creating and starting of process instances. All these PPoIs are directly related to MPoIs in the given MSC specification.

GANTT diagrams represent the state transitions of measured processes with respect to a common time. The application of GANTT for creating time-state-diagrams depends on the assignments of measured events to significant system states. Because states are defined in an MSC by using the keyword condition and events are defined by MISS while generating the instrumentation description, GANTT configuration files can be produced by MISS as well.

Besides behavior-oriented event trace analysis, MISS also supports statistics-oriented analysis using TRCSTAT which computes event frequencies and durations between events. To calculate message run times, events which represent the MPoIs for sending and receiving messages must be known; to compute timer expiration times MPoIs indicating the setting, resetting and expiring of the timer must be provided. The knowledge which events are necessary for calculating these values is contained in the MSC at corresponding MPoIs. Thus, TRCSTAT configuration files may be derived from an MSC too.

\section{CASE STUDY: MONITORING THE INRES PROTOCOL}

In this section we introduce the INRES protocol [Hog89] for discussing the main advantages of MSC-based monitoring for performance evaluation of SDL systems compared to conventional monitoring approaches. INRES offers a reliable, connection-oriented, and unsymmetrical service between two protocol entities called Initiator and Responder via its two service access points ISAPini and ISAPres. For data transmission INRES uses an unreliable, connectionless, and symmetric Medium service (see Figure 3). The protocol data units (PDUs) as well as the service primitives (SPs) of the INRES protocol are summarized in Table 1.

INRES is a three-phase protocol and supports a secure connection establishment between Initiator and Responder processes, an acknowledged data exchange and a final disconnection. During the connection establishment and the data exchange, the Initiator sets a timer directly after sending either a CR or a DT PDU. If the timer is adjusted too short a retransmission occurs and the communication will be delayed. In this situation, monitoring facilities are valuable in finding the reason of the delayed communication behavior.

Assuming a correct Medium service performance monitoring can be restricted to the INRES protocol instances exclusively. To prepare the specification-driven monitoring we choose an MSC specifying the communication behavior between the Initiator and Responder entities during connection establishment and data exchange, thereby neglecting all details concerning the Medium service. This MSC is shown in Figure 4 where the setting, resetting, and expiring behavior of the Initiator timer after sending each CR and DT PDU is of special interest. 


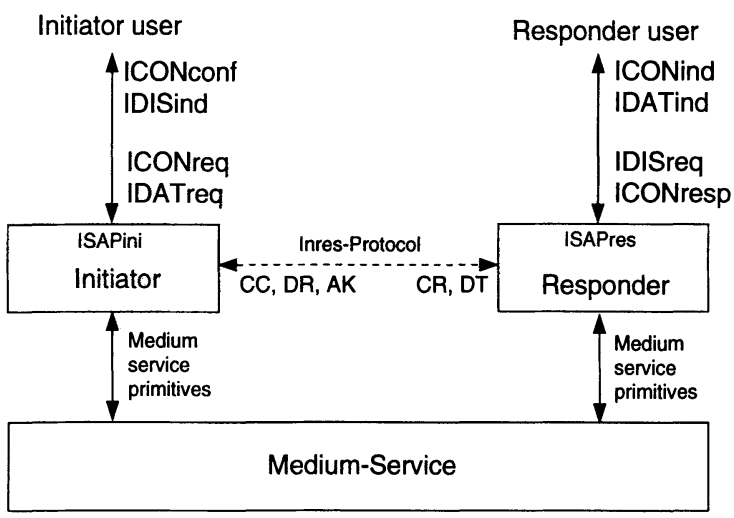

Figure 3 The INRES Protocol [Hog89].

\begin{tabular}{ll}
\hline PDU & \multicolumn{1}{c}{ Meaning } \\
\hline $\mathrm{CR}$ & connection request \\
\hline $\mathrm{CC}$ & connection confirmation \\
\hline $\mathrm{DT}^{\dagger}$ & data transfer \\
\hline $\mathrm{AK}^{\ddagger}$ & acknowledgement \\
\hline $\mathrm{DR}$ & disconnection request \\
\hline
\end{tabular}

\begin{tabular}{lll}
\hline \multicolumn{1}{c}{ SP } & \multicolumn{1}{c}{ Meaning } & PDU \\
\hline ICONreq & connection request & $\mathrm{CR}$ \\
\hline ICONind & connection indication & $\mathrm{CR}$ \\
\hline ICONresp & connection response & $\mathrm{CC}$ \\
\hline ICONconf & connection confirmation & $\mathrm{CC}$ \\
\hline IDATreq & data request & $\mathrm{DT}$ \\
\hline IDATind & data indication & $\mathrm{DT}$ \\
\hline IDISreq & disconnection request & $\mathrm{DR}$ \\
\hline IDISind & disconnection indication & $\mathrm{DR}$ \\
\hline
\end{tabular}

Table 1: INRES Protocol Data Units and Service Primitives

MISS is using this MSC for creating an appropriate instrumentation description (see Figure 1). For each specified state, i.e. Disconnected, Waiting, Connected and Sending as well as for each received and sent SP and PDU defined by the MSC, AICOS instrumentation statements are generated to carry out the instrumentation of the $\mathrm{C}$ code. The following representative extract of an instrumentation description describes the instrumentation for sending a CR PDU:

\footnotetext{
† Parameters of the DT PDU are a sequence number and the service data unit.
}

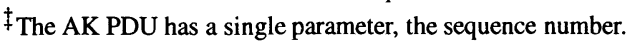




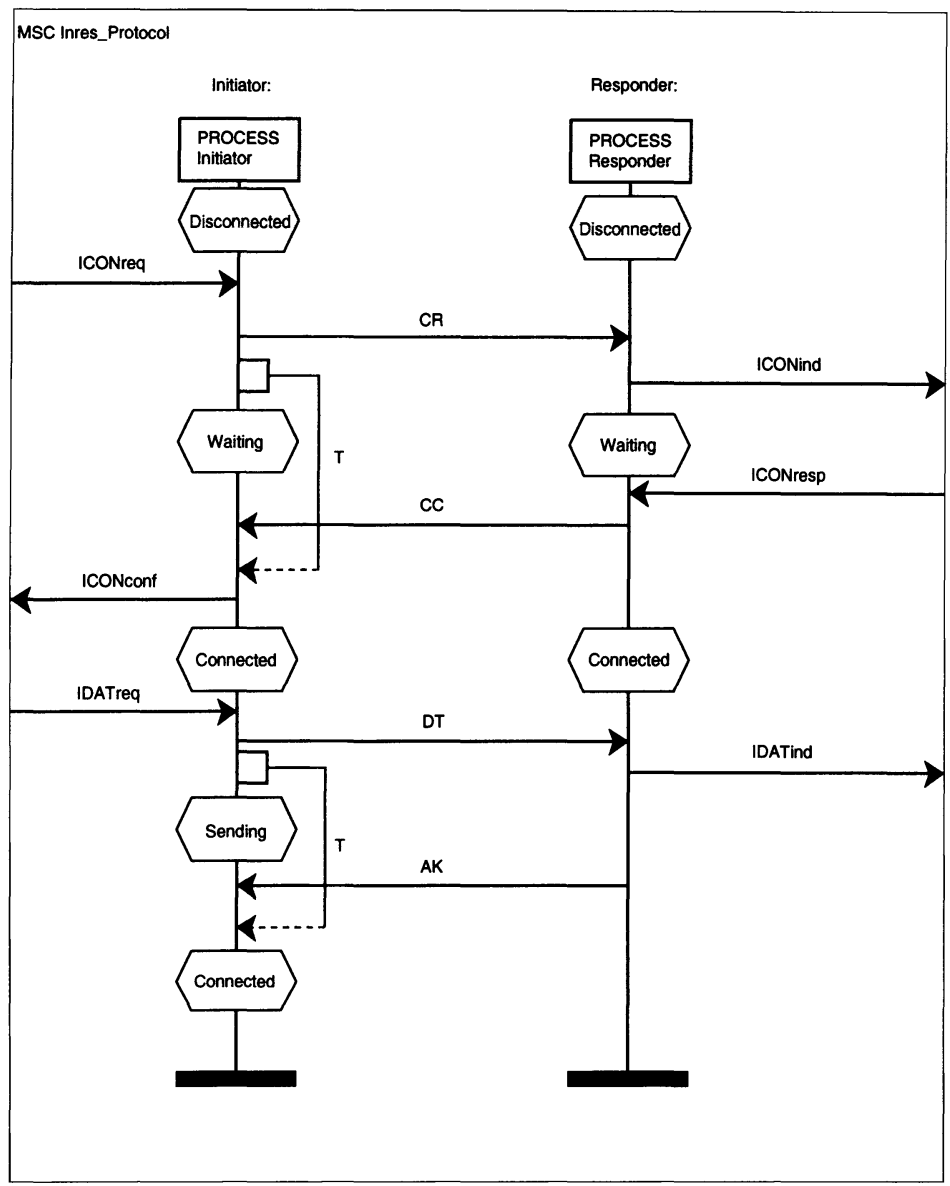

Figure 4 MSC used for monitoring the INRES protocol.

The first line indicates the event name (OUT_Cr) which is automatically generated by MISS. The prefixes OUT_ and IN _ are used to denote outgoing and incoming events, respectively. In the second line the AICOS command \#BEFOREANNOTATEDCALL indicates the insertion of the statement trace_2 (14，P->Self.GlobalNodeNr) ; directly before 


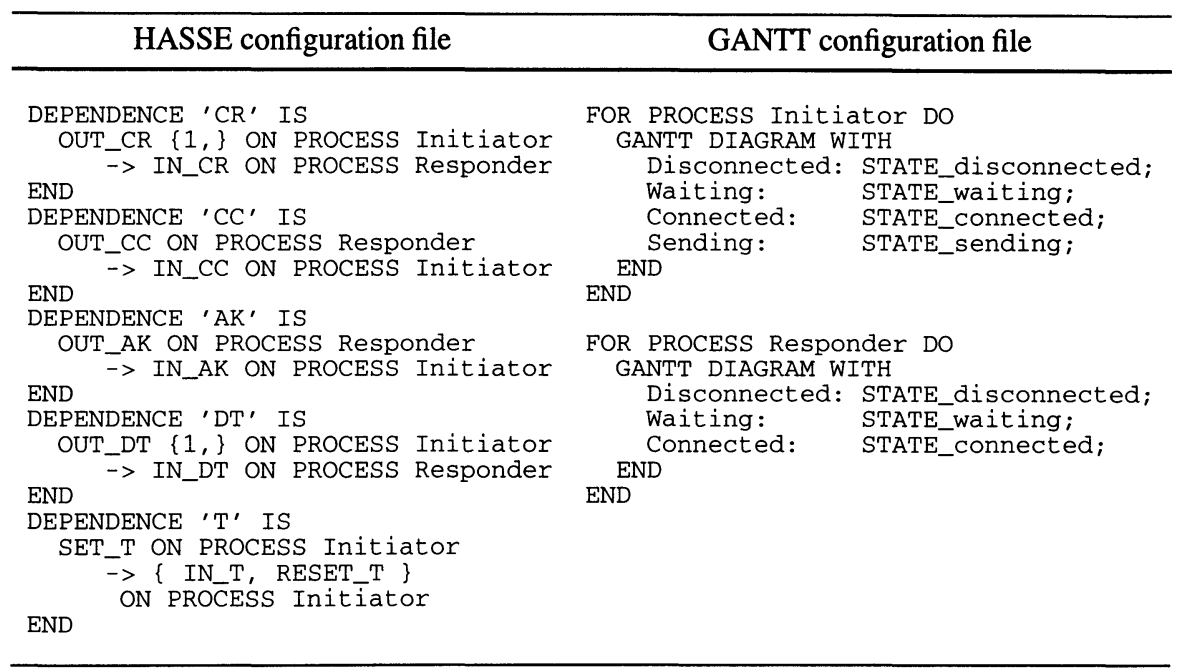

Table 2 HASSE and GANTT configuration file derived from the MSC ${ }^{\S}$.

the first call of the function SDL_Output and after the occurrence of an annotation / * " 6 Connectioncreate $130100 "$ */ in file Initiator.c. SDT annotations are unambiguous because they contain the X-and Y-coordinates of the corresponding SDL elements in the SDL/GR specification. Thus, there exists a one-to-one mapping between the SDL specification and its annotations. Instrumentation statements for other MPoIs given in the MSC of Figure 4 will be built in an analogous way.

After the automatic compilation by SDT the instrumented INRES protocol was executed on two SUN Sparc workstations interconnected via an IEEE802.3 LAN. The local clocks are synchronized using the network time protocol NTP [Mil94] which guarantees a common time base with a sufficient accuracy of $1 \mathrm{~ms}$. This enables the merge of the local event traces into a single global one with events ordered according to increasing time stamps.

MISS also generates configuration files for the various SIMPLE event trace analysis tools. Table 2 shows the derived configuration files for GANTT and HASSE, words in upper-case letters are keywords of the configuration languages.

The HASSE configuration file contains a causal dependence for each PDU exchanged between the Initiator and Responder entities plus one dependence describing the causal relationship between the timer events. Each dependence consists of a causing event on the left hand side of the arrow $(->)$ and a dependent event on its right hand side. The event name

§Events representing state entries or exits are prefixed with STATE_. For timers the prefixes SET_RESET_, and IN_ for setting, resetting or expiring of the timer are used. 


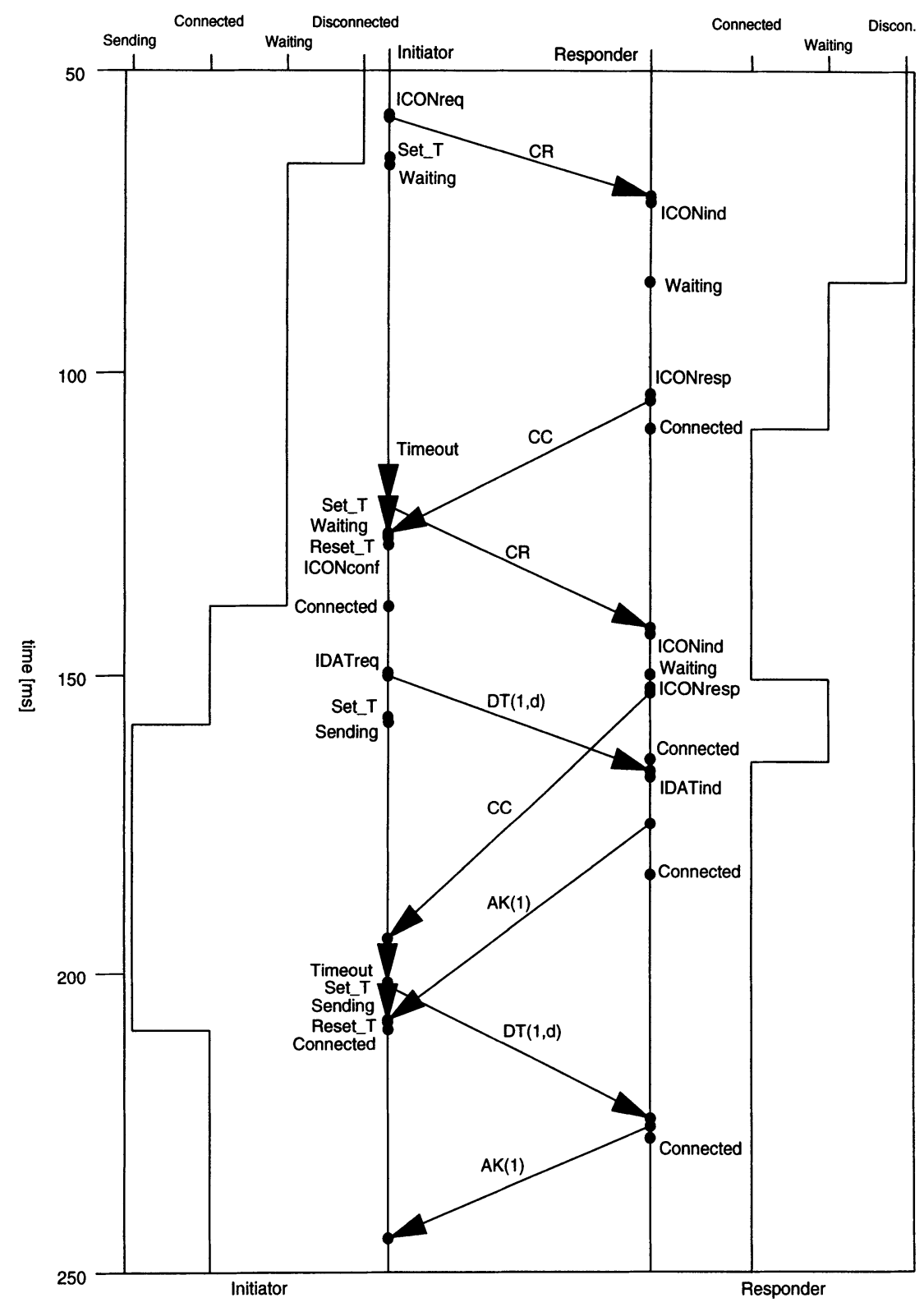

Figure 5 Combined HASSE and GANTT diagram. 
together with the process where the event occurs are given. In the GANTT configuration file two time-state-diagrams are specified. The diagram for the Initiator consists of the four states Disconnected, Waiting, Connected and Sending, whereas in the Responder diagram the Sending state is missing. The events associated with the different states are given behind the state names separated by a colon.

Figure 5 shows the result of analyzing the measured event trace with GANTT, respectively HASSE, and using the configuration files of Table 2. Here, the output of both tools are visualized in one single diagram: the HASSE part is drawn in the middle while on the left and right GANTT time-state-diagrams of the Initiator and Responder are shown, respectively. The HASSE diagram reveals that two CR PDUs are sent by the Initiator before the connection is established successfully. Shortly, after the first CR PDU is sent the timer T is set to an initial value of 60 ms and expires after this time period. This causes a second CR PDU to be sent by the Initiator because the expected Responder CC PDU arrives too late. Obviously, the timer's expiration time is adjusted too short. Despite of the late arrival of the first CC PDU, the Initiator consumes it, notifies a successful connection establishment to the user (ICONconf event), and changes into the Connected state. While connected a user IDATreq request causes the Initiator to send a DT PDU to the Responder, to set a timer, and to turn into the Sending state while waiting for a Responder AK PDU. Again, the timer expires before the Responder AK PDU arrives which causes the Initiator to repeat sending the first DT PDU. In contrast to the receipt of the first DT PDU where the Responder indicates the exchange of user data with an IDATind, the Responder ignores doubled data $\$$ by sending a repeated AK PDU to the Initiator.

Comparing the given MSC in Figure 4 with the event trace evaluation results presented in Figure 5 explains the delayed communication behavior of the INRES protocol. The obvious conclusion from Figure 5 is to enlarge the timer value in order to save Medium bandwidth and to speedup the communication between Initiator and Responder entities. In general, the presented method of MSC-based monitoring of SDL systems gives us a tool to adjust time-sensitive parameters of complex parallel and distributed systems.

\section{CONCLUDING REMARKS}

In this paper we have introduced a new method which is suitable for monitoring complex realworld applications specified with SDL: MSC-based monitoring. The main advantages of the method can be summarized as follows:

- Event definition, the hardest job when dealing with monitoring, is supported by the MSC scenario in a systematic way.

- MSC-based event definitions enables automatic source code instrumentation.

- MSC scenarios focus on application-specific PoIs for performance evaluation and neglect surplus details.

- Interdependences provided in MSC scenarios support automatic generation of meaningful and application-specific monitoring experiments.

TThe Responder recognizes doubled data by means of the sequence number transmitted in each DT and AK PDU. 
- Only fully automatic specification-driven monitoring allows the user-friendly integration of monitoring tools in the SDL/MSC software engineering cycle.

- MSC-based techniques can be applied for analytical and simulative performance studies of SDL specified systems which is outlined in [Dul94] and is the topic of further research.

Note, that the method described so far may also be applied in a cyclic and hierarchical way. Suppose the performance results of an MSC-based event trace analysis indicate a strange behavior in parts of the SuS. The interpretation of the performance results will require a hierarchical refinement of the MSC-based monitoring to get deeper insight in the identified parts of the SDL system. It will lead to new measurements and detailed performance results concerning the observation of the refined parts of the SDL system. The hierarchical refinement is supported by both, the hierarchical structure of the MSCs which may consist of sub-MSCs, and by utilizing detailed knowledge of the SDL system itself. This cyclic and hierarchical approach serves for iteratively improving an SDL specification, e.g. to eliminate a bottleneck found.

The proposed method of specification-driven monitoring is also suitable to examine whether the automatic generation of $\mathrm{C}$ code from a given SDL specification produces an implementiation which meets a required efficiency.

Besides, the statistical values calculated with MSC-based monitoring can be applied to attribute a given MSC with realistic parameters in order to carry out performance simulations for performance prediction of alternative solutions of the system under study.

\section{REFERENCES}

[BB89] T. Bolognesi and E. Brinksma. Introduction to the ISO Specification Language LOTOS. In P.H.J. van Eijk, C.A. Vissers, and M. Diaz, editors, The Formal Description Technique LOTOS, pages 23-73, Amsterdam, 1989. North-Holland.

[BR93] T. Bemmerl and B. Ries. Programming Tools for Distributed Multiprocessor Computing Environments. International Journal of High Speed Computing, 5(4):595-615, December 1993.

[CCI92] CCITT. Recommendation Z.100: Specification and Description Language SDL, Blue Book. ITU General Secreteriat - Sales Section, Place des Nations, CH-1211 Geneva 20, 1992.

[DHK ${ }^{+93]}$ P. Dauphin, F. Hartleb, M. Kienow, V. Mertsiotakis, and A. Quick. PEPP: Performance Evaluation of Parallel Programs - User's Guide - Version 3.3. Technical Report 17/93, Universität Erlangen-Nürnberg, IMMD VII, September 1993.

[DKQ92] P. Dauphin, M. Kienow, and A. Quick. Model-driven Validation of Parallel Programs Based on Event Traces. In Topham, Ibbett, and Bemmerl, editors, Proceedings of the "Working Conference on Programming Environments for Parallel Computing”, Edinburgh 6-8 April, pages 107-125, 1992.

[Dul94] W. Dulz. Leistungsbewertung von SDL/MSC-spezifizierten Protokollen . In GI/ITGArbeitsgespräch "Modellierungs- und Bewertungstools 94", Uni Dortmund, 1994.

[GEO94] GEODE. Geode - Technical Presentation . VERILOG SA, France, 1994. 
[HE91] M.T. Heath and J. A. Etheridge. ParaGraph: A Tool for Visualizing Performance of Parallel Programs. Technical report, Oak Ridge National Laboratory, Tennessee, November 1991.

[HKM $\left.{ }^{+} 94\right]$ R. Hofmann, R. Klar, B. Mohr, A. Quick, and M. Siegle. Distributed Performance Monitoring: Methods, Tools, and Applications. IEEE Transactions on Parallel and Distributed Systems, 5(6):585-598, June 1994.

[Hog89] Dieter Hogrefe. Estelle, LOTOS und SDL. Springer, Berlin, 1989.

[HQ93] F. Hartleb and A. Quick. Performance Evaluation of Parallel Programms - Modeling and Monitoring with the Tool PEPP. In B. Walke and O. Spaniol, editors, Proceedings der 7. GI-ITG Fachtagung "Messung, Modellierung und Bewertung von Rechen- und Kommunikationssystemen “, Aachen, 21.-23. Spetember 1993, pages 51-63. Informatik Aktuell, Springer, 1993.

[IT94] ITU-T. Recommendation Z.100: Appendices I and II. International Telecommunication Union - Telecommunication Standardization Sector, Place des Nations, CH-1211 Geneva 20, 1994.

[Liu89] Ming T. Liu. Protocol engineering. In Marshall C. Yovits, editor, Advances in Computers, volume 29, pages 79-195. Academic Press Inc., Boston, 1989.

[MHJ91] A.D. Malony, D.H. Hammerslag, and D.J. Jablonowski. Traceview: A Trace Visualization Tool. IEEE Software, September 1991.

[Mil94] D.L. Mills. Improved algorithms for synchronizing computer network clocks. Computer Communication Review, 24(4):317-327, October 1994.

[Moh91] B. Mohr. SIMPLE: a Performance Evaluation Tool Environment for Parallel and Distributed Systems. In A. Bode, editor, Distributed Memory Computing, 2nd European Conference, EDMCC2, pages 80-89, Munich, Germany, April 1991. Springer, Berlin, LNCS 487.

[Moh92] B. Mohr. SIMPLE - User's Guide Version 5.3.. Technical Report 3/92, Universität Erlangen-Nürnberg, IMMD VII, März 1992.

[Qui93] A. Quick. The $M^{2}-C y c l e:$ Model-driven Monitoring for the Evaluation of Parallel Programs (in German). $\mathrm{PhD}$ thesis, University of Erlangen-Nürnberg, 1993. to be published.

[Te193] TeleLOGIC. SDT Version 2.3 Reference Manual, Volume 1 and 2, SDT Users Guide, SDT Technical Presentation. TeleLOGIC, Malmö, 1993.

[TOP94] Project TOPIC. Integration of the Toolset Prototypes (V1) . Technical report, CEC Deliverable Number R2088/GMD/SEM/DS/L/013/b5, March 26, 1994, 1994. 